\title{
Two-dimensional Matched Filtering Impact on Signal to Noise Ratio
}

\author{
Feng Jibin \\ School of Information and Electronics \\ Beijing Institute of Technology \\ Beijing, China \\ e-mail: jibin5453268@126.com

\section{Zhang Kai} \\ School of Information and Electronics \\ Beijing Institute of Technology \\ Beijing, China \\ e-mail: zhangkai3325@163.com
}

\author{
Li Wen \\ School of Information and Electronics \\ Beijing Institute of Technology \\ Beijing, China \\ e-mail: 195589887@qq.com
}

\begin{abstract}
Synthetic aperture radar (synthetic aperture radar, SAR) use a two-dimensional matched filter to improve the signal to noise ratio, but the signal-to-noise ratio (SNR) gain of synthetic aperture radar (SAR) is controversial. The traditional method analyzes the SAR signal-to-noise ratio based on pulse compression and coherent integration, but it will have different calculation results. In this paper, in connection with the characteristics of the SAR digital signal processing, the SNR gain of the two-dimensional matched filter is derived to solve the contentious issue of SAR signal-to-noise ratio, which is based on the assumption of the band-limited white noise in the digital domain, and the impact of matched filtering on SNR is analyzed and the optimized design criteria of sampling rate is presented. This conclusion of analysis is verified by simulations, which provides a theoretical basis for the SAR system design.
\end{abstract}

Keywords-synthetic aperture radar (SAR); SNR; pulse compression; optimized design; matched filter

\section{INTRODUCTION}

Synthetic aperture radar (synthetic aperture radar, SAR) combine the pulse compression and synthetic aperture radar technology ${ }^{[1,2]}$, which uses pulse compression technology to achieve high resolution in the distance dimension, The azimuth dimension high resolution rely on coherent processing of the long synthetic aperture. In the pulse compression radar, the improvement for SNR ratio is presented with the compressed ratio form of the pre-compression pulse width

$\tau_{i}$ and the compressed pulse width $\tau_{0}$, it is easy to know that the signal-to-noise gain for SAR distance of the pulse compression processing is the product of time-width and band-width ${ }^{[3]}$. In using the synthetic aperture forming techniques to achieve the required azimuth resolution of the radar, because of the accumulation of a large number of pulses, so the processing to the azimuth signal is also increased by a improvement factor of SNR.

SAR coherently accumulate $n$ echo signals within a synthetic aperture time $T_{a}$,from the perspective of coherent integration, the azimuth processing gain of SNR is the accumulated number of pulses in azimuth, which is equal to the product of the pulse repetition frequency and the time required to form a synthetic aperture ${ }^{[4]}$; however, from the perspective of matched filtering, SAR azimuth processing is also equivalent to the matched filtering process, then the SNR gain is the product of azimuth Doppler signal bandwidth and the accumulation time ${ }^{[5]}$. From this point, there are two calculation methods about SNR gain currently ${ }^{[6-8]}$, but the calculation results will be different. Therefore, the SAR SNR gains for a twodimensional signal processing need be analyzed.

In this paper, Aiming at the characteristics of SAR signal processing, the SNR gain formula for digital matched filter is derived from a continuous signal matched filtering process, then the two-dimensional SNR gain after SAR matched filtering process is mathematical analyzed, and this paper verify the theoretical analysis through simulation results finally, solving the disputed issue of SAR SNR gain.

\section{THE SNR GAIN OF DIGITAL MATCHED FILTER}

In the process of SAR imaging, the method of twodimensional matched filtering can improve the signal-tonoise ratio ${ }^{[10,11]}$, but there is debating about the SNR gain. For example on the SNR gain of SAR radar equation, Azimuth matched filter SNR gain sometimes use the product of the Doppler bandwidth and the accumulation time, while others are the product of the pulse repetition frequency and accumulation time. According to the literature $^{[12,13]}$, it is easy to know the matched filter is the output signal-to-noise largest ratio of the filter under the white noise condition, but the SNR gain analysis is primarily based on continuous time signal, and on the formation of the SAR echo signal, The form of azimuth signal has been discrete based on the assumption of "stop and go". in addition, in the process of project implementation, it tend to adopt the way of digital signal processing to realize two-dimensional matched filtering, so it is necessary to analyze the SNR gain after discrete matched filtering ${ }^{[14]}$.So there has discussion about one- 
dimensional discrete time signals for the improvement of signal-to-noise ratio.

Suppose the input signal of matched filter is $s(t)$, and its corresponding frequency spectrum can be represented as:

$$
S(f)=\left\{\begin{array}{cc}
E_{0} & f_{c}-B_{s} / 2<f<f_{c}+B_{s} / 2 \\
0 & \text { else }
\end{array}\right.
$$

Where $E_{0}$ is the amplitude spectrum of the signal, $B_{s}$ is the signal bandwidth, $f_{c}$ is the Center frequency.

Suppose the input random noise is the smooth bandlimited white noise, and its power spectrum can be represented as:

$$
N(f)= \begin{cases}N_{0} & -B_{n} / 2<f<B_{n} / 2 \\ 0 & \text { else }\end{cases}
$$

Where $N_{0}$ is the power spectral density, $B_{n}$ is noise bandwidth, so the input noise power is:

$$
P_{\text {in }}=\int_{-\infty}^{+\infty} N(f) \mathrm{d} f=N_{0} B_{n}
$$

Then, after the matched filter $H(\omega)=S^{*}(\omega)$, the matched filter SNR gain can be expressed as:

$$
G=\frac{\mathrm{SNR}_{\mathrm{o}}}{\mathrm{SNR}_{\mathrm{i}}}=\frac{E_{p}^{2} / \int_{-\infty}^{+\infty} N(f)|H(f)|^{2} \mathrm{~d} f}{E_{p} / T_{p} / \int_{-\infty}^{+\infty} N(f) \mathrm{d} f}
$$

Where $\mathrm{SNR}_{\mathrm{O}}$ is the output signal-to-noise ratio, $\mathrm{SNR}_{\mathrm{i}}$ is the input signal-to-noise ratio, The energy of the signal $E_{p}$ can be represented as:

$$
E_{p}=\int_{-\infty}^{+\infty}|S(f)|^{2} \mathrm{~d} f
$$

The SNR gain of the formula (4) can be expressed as following expression for the condition of continuous time signals:

$$
G=\frac{E_{p} T_{p} \int_{-\infty}^{+\infty} N(f) \mathrm{d} f}{\int_{-\infty}^{+\infty} N(f)|H(f)|^{2} \mathrm{~d} f}=\frac{E_{p} T_{p} N_{0} B_{n}}{N_{0} E_{p}}=B_{n} T_{p} \text { (6) }
$$

After the input continuous random noise digital sampling, white noise becomes colored noise, the noise power spectrum will change along with the change of sampling rate, discrete SNR gain can be obtained:

$G_{m}=\frac{E_{p} T_{p} \int_{-\infty}^{+\infty} N(f) \mathrm{d} f}{\int_{-\infty}^{+\infty} N(f)|H(f)|^{2} \mathrm{~d} f}=\frac{B_{S} T_{p} \sum_{n=-\infty}^{+\infty} \int_{-f_{S} / 2}^{f_{S} / 2} N\left(f-n f_{s}\right) \mathrm{d} f}{\sum_{n=-\infty}^{+\infty} \int_{-B_{S} / 2}^{B_{S} / 2} N\left(f-n f_{S}\right) \mathrm{d} f}$

Where $f_{s}$ is the sampling rate of the signal. Molecular summation item of the formula (7) represents the input noise power after sampling, and The denominator summation item represents output noise power after matched filtered, make:

$$
\begin{gathered}
P_{\text {in }}=\sum_{n=-\infty}^{+\infty} \int_{-f_{S} / 2}^{f_{S} / 2} N\left(f-n f_{S}\right) \mathrm{d} f \\
P_{\text {on }}=\sum_{n=-\infty}^{+\infty} \int_{-B_{S} / 2}^{B_{S} / 2} N\left(f-n f_{S}\right) \mathrm{d} f
\end{gathered}
$$

Suppose $x=\left(f-n f_{s}\right) / f_{s}$, The input noise power is:

$$
P_{i n}=\sum_{n=-\infty}^{+\infty} \int_{n-1 / 2}^{n+1 / 2} f_{s} N\left(x f_{s}\right) \mathrm{d} x
$$

Where:

$$
N\left(x f_{s}\right)= \begin{cases}N_{0} & -B_{n} / 2 f_{s}<x<B_{n} / 2 f_{s} \\ 0 & \text { else }\end{cases}
$$

In the same way, the available output noise power is:

$$
P_{\text {on }}=\sum_{n=-\infty}^{+\infty} \int_{n-B_{S}}^{n+B_{S} / 2 f_{S}} f_{S} N\left(x f_{S}\right) \mathrm{d} x
$$

The following is mainly for different noise bandwidth $B_{n}$, the sampling rate $f_{s}$ and the bandwidth of the signal $B_{s}$, analyzing the gain of the signal to noise ratio:

(1) if $B_{n} / 2 f_{s} \leq 1 / 2$, then $f_{s} \geq B_{n} \geq B_{s}$

$$
\begin{array}{r}
P_{\text {in }}=\sum_{n=-\infty}^{+\infty} \int_{n-1 / 2}^{n+1 / 2} f_{s} N\left(x f_{s}\right) \mathrm{d} x=N_{0} B_{n} \\
P_{\text {on }}=\sum_{n=-\infty}^{+\infty} \int_{n-B_{S} / 2 f_{S}}^{n+B_{S} / 2 f_{s}} f_{S}\left(x f_{s}\right) \mathrm{d} x=N_{0} B_{s}
\end{array}
$$

Thus you can get the SNR gain is:

$$
G_{m}=\frac{B_{s} T_{p} \sum_{n=-\infty}^{+\infty} \int_{-f_{s} / 2}^{f_{s} / 2} N\left(f-n f_{s}\right) \mathrm{d} f}{\sum_{n=-\infty}^{+\infty} \int_{-B_{S} / 2}^{B_{S} / 2} N\left(f-n f_{s}\right) \mathrm{d} f}=B_{n} T_{p}
$$

From the formula can be seen, when meeting the relation of condition (1), the signal-to-noise ratio after the matched filtering gain is the product of noise bandwidth and time-width of signal, filter bandwidth and signal bandwidth is usually approximately equal in radar receiving[14], we can think SNR gain as the product of the time-width and signal bandwidth. However, when the radar system needs to receive a variety of different bandwidth of the signal, if the coverage of signal bandwidth is very large, in order to reduce hardware resources and reduce the complexity of the system, the receiver filter often require the biggest bandwidth according to the signal. When receiving small signal bandwidth, noise bandwidth will be greater than the signal bandwidth, so this paper need to analysis the different conditions SNR gain.

(2) if $B_{n} / 2 f_{s}>1 / 2$, then $B_{n} \geq f_{s} \geq B_{s}$

Suppose $p=\left[B_{n} / 2 f_{s}+1 / 2\right]$, where $[\bullet]$ is presented as rounded part. $q=B_{n} / 2 f_{s}+1 / 2-p$, The formula (15) the denominator summation item contains $2 p+1$ integral interval, including $2 p-1$ complete integral and two noncompleted integral interval.

$$
\begin{aligned}
& P_{\text {in }}=\sum_{n=-\infty}^{+\infty} \int_{n-1 / 2}^{n+1 / 2} f_{S} N\left(x f_{s}\right) \mathrm{d} x=f_{S} N_{0}(2 p-1+2 q)=N_{0} B_{n} \\
& P_{\text {on }}=(2 p-1) N_{0} B_{S}+\int_{p-B_{S}}^{p+B_{S} / 2 f_{S}} f_{S} N\left(x f_{s}\right) \mathrm{d} x
\end{aligned}
$$

Where, the output noise power of the formula (17) needs the relations between the noise bandwidth, signal bandwidth, and sampling rate, according to this, the following which can be divided into three conditions are discussed:

$$
\text { (1)if } q \geq \frac{1}{2}+\frac{B_{s}}{2 f_{s}}, \text { then }
$$




$$
\begin{aligned}
& P_{\text {on }}=(2 p-1) N_{0} B_{s}+2 N_{0} B_{s}=(2 p+1) N_{0} B_{s} \\
& \text { (2)if } q \leq \frac{1}{2}-\frac{B_{s}}{2 f_{S}} \text {, then } \\
& \qquad P_{\text {on }}=(2 p-1) N_{0} B_{s} \\
& \text { (3) if } \frac{1}{2}-\frac{B_{s}}{2 f_{s}} \leq q \leq \frac{1}{2}+\frac{B_{s}}{2 f_{s}} \text {, then } \\
& \qquad P_{o n}=N_{0}\left[B_{n}-2 p\left(f_{s}-B_{s}\right)\right]
\end{aligned}
$$

Thus you can get the SNR gain of the matched filtering after the discrete sampling:

$$
G_{m}=\left\{\begin{array}{lr}
\frac{1}{2 p+1} B_{n} T_{p} & q \geq \frac{1}{2}+\frac{B_{s}}{2 f_{s}} \\
\frac{1}{2 p-1} B_{n} T_{p} & q \leq \frac{1}{2}-\frac{B_{s}}{2 f_{s}} \\
B_{n} T_{p} & \text { else }
\end{array}\right.
$$

From the above analysis can be seen, the signal-tonoise ratio of the digital matched filter gain is decided by the relative relations noise bandwidth $B_{n}$, sampling rate $f_{S}$ and signal bandwidth $B_{s}$. from the formula (21) can be seen, when $f_{s}=B_{n} / 2 n-1, n=0,1,2 \mathrm{~L}, G_{m}=f_{s} T_{p}$, the SNR gain will be reduced by using the low sampling rate of noise samples the signal,. When $f_{s}=\left(B_{n}+B_{s}\right) / 2(n+1) \quad n=0,1,2 \mathrm{~L}$, SNR gain periodic maximum can be achieved, then $G_{m}=\left[2(n+1) f_{s}+B_{s}\right] T_{p} \quad$, when $\quad n=0 \quad$ and $f_{s}=\left(B_{n}+B_{s}\right) / 2$ are met, the SNR gain can reach maximum value.

\section{THE SNR GAIN OF TWO-DIMENSIONAL MATCHED FILTERING}

SAR imaging process is essentially a twodimensional matched filtering process, in order to handle and implementation easily, it is generally achieving twodimensional matched filtering in digital domain through data collection, According to the analysis conclusion, the signal-to-noise ratio of the distance to the matched filtering gain is:

$$
G_{r}=\frac{B_{s} T p \sum_{n=-\infty}^{+\infty} \int_{-f_{S} / 2}^{f_{S} / 2} N\left(f-n f_{s}\right) \mathrm{d} f}{\sum_{n=-\infty}^{+\infty} \int_{-B_{S} / 2}^{B_{S} / 2} N\left(f-n f_{s}\right) \mathrm{d} f}
$$

Similarly the azimuth matched filter SNR gain is:

$$
G_{a}=\frac{B_{d} T_{s} \sum_{n=-\infty}^{+\infty} \int_{-F_{r} / 2}^{F_{r} / 2} N_{L}\left(f-n F_{r}\right) \mathrm{d} f}{\sum_{n=-\infty}^{+\infty} \int_{-B}^{B} d_{d} / 2 N_{L}\left(f-n F_{r}\right) \mathrm{d} f}
$$

Where $F_{r}$ is the pulse repetition frequency or the azimuth sampling rate; $B_{d}$ is the azimuth Doppler bandwidth, The
SNR gain of the two-dimensional matched filtering can be expressed as:

$$
\begin{gathered}
G_{s}=G_{r} G_{a}=\frac{B_{s} T_{p} \sum_{n=-\infty}^{+\infty} \int_{-f_{s} / 2}^{f_{S} / 2} N\left(f-n f_{s}\right) \mathrm{d} f}{\sum_{n=-\infty}^{+\infty} \int_{-B_{S} / 2}^{B_{S} / 2} N\left(f-n f_{s}\right) \mathrm{d} f} \times \\
\frac{B_{d} T_{s} \sum_{n=-\infty}^{+\infty} \int_{-F_{r} / 2}^{F_{r} / 2} N_{L}\left(f-n F_{r}\right) \mathrm{d} f}{\sum_{n=-\infty}^{+\infty} \int_{-B}^{B} d_{d}^{/ 2} N_{L}\left(f-n F_{r}\right) \mathrm{d} f}
\end{gathered}
$$

The prior to azimuth matched filtering process, the input noise power is the output noise power after distance processing, according to the previous derivation, the noise power is kept constant after sampling, then:

$$
\sum_{n=-\infty}^{+\infty} \int_{-B_{S} / 2}^{B_{S} / 2} N\left(f-n f_{S}\right) \mathrm{d} f=\sum_{n=-\infty}^{+\infty} \int_{-F_{r} / 2}^{F_{r} / 2} N_{L}\left(f-n F_{r}\right) \mathrm{d} f
$$

So can get:

$$
G_{s}=\frac{B_{s} T_{p} B_{d} T_{s} \sum_{n=-\infty}^{+\infty} \int_{-f_{s} / 2}^{f_{s} / 2} N\left(f-n f_{s}\right) \mathrm{d} f}{\sum_{n=-\infty}^{+\infty} \int_{-B_{d} / 2}^{B} / 2 N_{L}(f-n P R F) \mathrm{d} f}
$$

then:

$$
G_{s}=\frac{B_{s} T_{p} B_{d} T_{s} N_{0} B_{n}}{\sum_{n=-\infty}^{+\infty} \int_{-B_{d} / 2}^{B} N_{L}\left(f-n F_{r}\right) \mathrm{d} f}
$$

when matching filter in azimuth, the analogy with the distance to the matched filter can be considered as input the noise bandwidth $B_{s}$, the sampling rate $F_{r}$, azimuth signal bandwidth $B_{d}$. The same $k=\left[B_{s} / 2 F_{r}+1 / 2\right] l=B_{s} / 2 F_{r}+1 / 2-k$ can be set. Digital matched filter according to the preceding conclusions derived found that the formula (27) in the denominator can be expressed as:

$$
\begin{aligned}
& \sum_{n=-\infty}^{+\infty} \int_{-B_{d}}^{B} d_{d}^{/ 2} / 2 N_{L}\left(f-n F_{r}\right) \mathrm{d} f= \\
& \left\{\begin{array}{lc}
(2 k+1) N_{0}{ }^{\prime}{ }^{B} & l \geq \frac{1}{2}+\frac{B_{d}}{2 F_{r}} \\
(2 k-1) N_{0}{ }^{\prime} B_{d} & l \leq \frac{1}{2}-\frac{B_{d}}{2 F_{r}} \\
N_{0}{ }^{\prime}\left(2 k B_{d}+2 l F_{r}-F_{r}\right) & \text { else }
\end{array}\right.
\end{aligned}
$$

Where, $N_{0}{ }^{\prime}$ is the equivalent noise power spectrum before azimuth processing, After the distance processing is completed, the noise bandwidth is limited within the signal bandwidth, the equivalent noise power spectrum is: 


$$
N_{0}^{\prime}=P_{o n} / B_{S}=\left\{\begin{array}{lr}
(2 p+1) N_{0} & q \geq \frac{1}{2}+\frac{B_{s}}{2 f_{s}} \\
(2 p-1) N_{0} & q \leq \frac{1}{2}-\frac{B_{s}}{2 f_{s}} \\
N_{0}\left[\frac{B_{n}}{B_{s}}-2 p\left(\frac{f_{s}}{B_{s}}-1\right)\right] & \text { else }
\end{array}\right.
$$

From the formula(27), (28) and (29) can be seen,the signal-to-noise ratio of the two-dimensional matched filtering gain are related with signal bandwidth, Dopplerbandwidth, noise bandwidth and $F_{r}$, In order to meet the requirements of the two dimensional resolution usually in SAR system design, the formula $B_{s}>F_{r} \geq B_{d}, k$ ? 1 is easy to satisfy, and the SNR gain can be approximate to:

$$
G_{s} \approx \frac{B_{s} T_{p} T_{s} B_{n}}{2 k} \approx \frac{B_{s} T_{p} T_{s} B_{n}}{B_{s} / F_{r}}=T_{p} T_{s} B_{n} F_{r}
$$

Therefore, the two-dimensional processing gain of the SAR can approximate thought as the following conditions, the processing gain of the distance is the product of the noise bandwidth and signal time width, Azimuth processing gain is the number of accumulated pulses. and the SAR SNR gain according to the formula (30) need be calculated precise under the condition of other specific parameters.

\section{SimUlation ANALYSIS}

In the system parameter conditions of the following table 1 , simulation on the signal-to-noise ratio gain of digital matched filtering processing.

\section{TABLE I. SYSTEM PARAMETERS}

\begin{tabular}{ll}
\hline Parameters name & value \\
\hline Signal bandwidth $B_{s}$ & $5 \mathrm{MHz}$ \\
\hline Sampling rate $f_{s}$ & $50 \mathrm{MHz}$ \\
\hline Pulse width $T_{p}$ & $10 \mu \mathrm{s}$ \\
\hline Accumulation time $T_{s}$ & $0.2 \mathrm{~s}$ \\
\hline The pulse repetition frequency $F_{r}$ & $2000 \mathrm{~Hz}$ \\
\hline
\end{tabular}

Adopting the method of theoretical calculation, matched filtering SNR gain and noise bandwidth relations can be achieved in Fig .1, from the Fig .1 we can see, when the noise bandwidth $2 n f_{s}+B_{s} \leq B_{n} \leq 2(n+1) f_{s}-B_{s}$ is met, SNR gain increase monotonously with the noise bandwidth; When the following formula $2(n+1) f_{s}-B_{s} \leq B_{n} \leq 2(n+1) f_{s}+B_{s}$ is met, the SNR gain monotonously decrease with noise bandwidth. Therefore, the signal-to-noise ratio of the matched filtering gain is relevant with noise bandwidth under the condition of the discrete band-limited white noise, it can present a cyclical change, and it can obtain the maximum periodically.

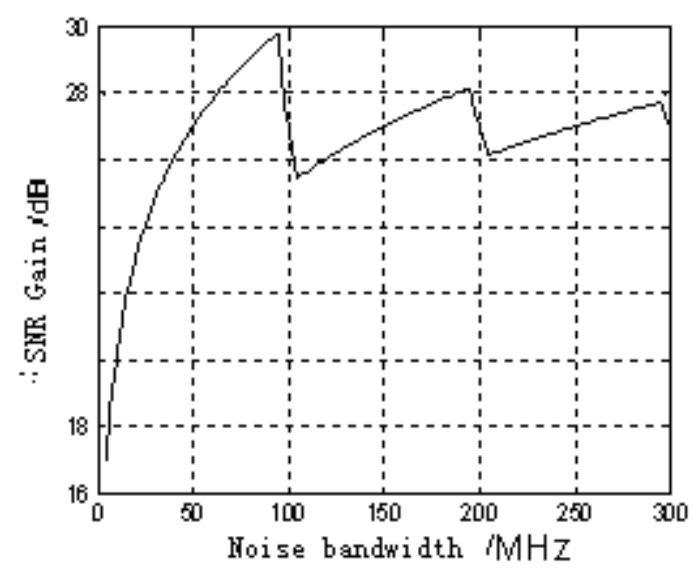

Figure 1. Relationship between the one-dimensional matched filter SNR gain and noise bandwidth

This paper uses the method of monte carlo simulation results which can verify the theoretical derivation, the signal-to-noise ratio of the matched filtering gain simulation results are shown in Fig .2 under the condition of test times for 5000 times, after many repeated trials, the SNR gain take the average of the many test results, table 2 is presented under the condition of different noise bandwidth, the matched filtering SNR gain simulation results can be contrast with the theoretical calculation. Because of the noise statistical characteristics, the theoretical value and the simulation results are basically consistent, so as to verify the validity of the theoretical derivation. when the noise bandwidth $B_{n}=95 \mathrm{MHz}, \mathrm{SNR}$ gain maximum, the relationship $B_{n}=2 f_{S}-B_{S}$ can be met at this time, the calculation result is consistent with the theory of formula (21). In addition, when the system noise bandwidth and signal bandwidth are fixed, output signalto-noise ratio of the system can achieve the optimal value by reasonably choosing the sampling rate, the optimal sampling rate can be designed as $f_{s}^{*}=\left(B_{n}+B_{s}\right) 2$,

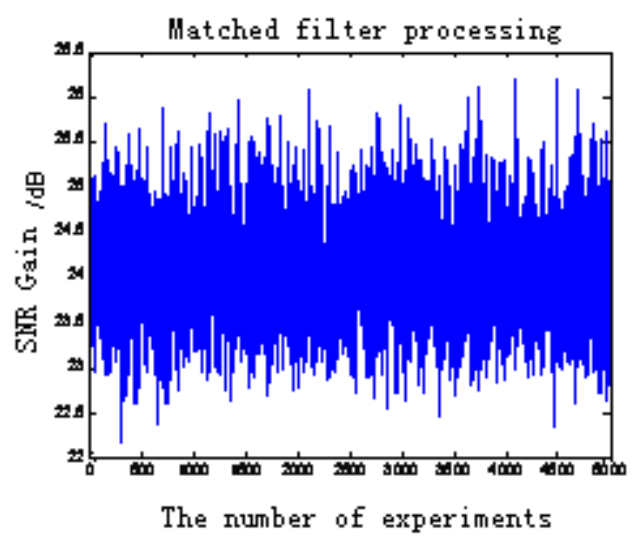

(a)Noise bandwidth $\mathrm{Bn}=25 \mathrm{MHz}$ 

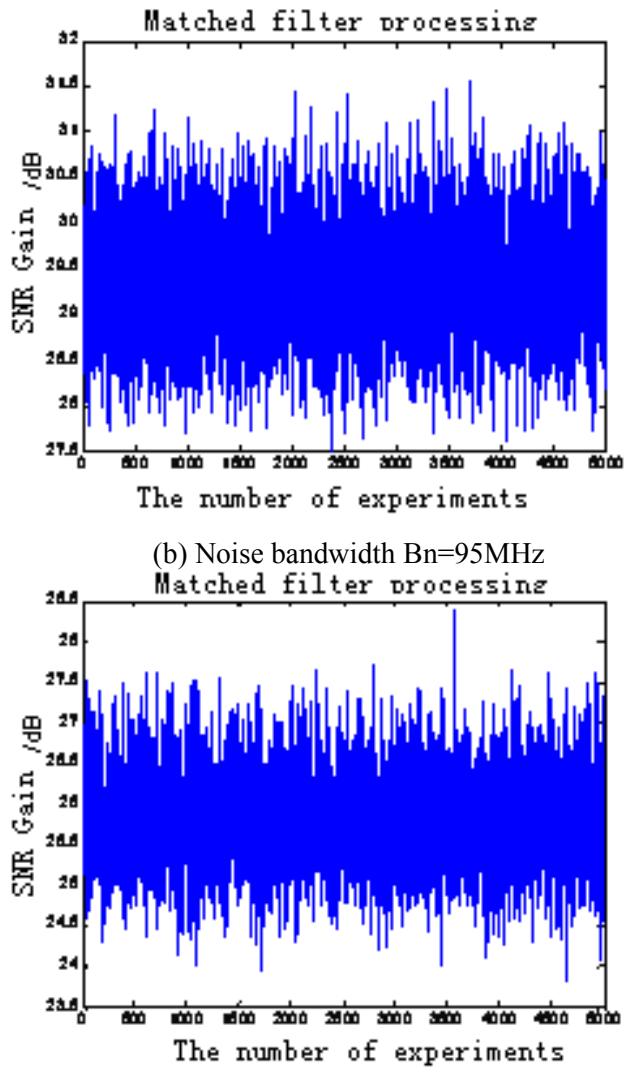

(c) Noise bandwidth $\mathrm{Bn}=105 \mathrm{MHz}$

Figure 2. the noise bandwidth SNR gain of different conditions

TABLE II. THE SNR GAIIN

\begin{tabular}{lll}
\hline $\begin{array}{l}\text { Noise-bandwidth } \\
\text { Bn }(\mathrm{MHz})\end{array}$ & $\begin{array}{l}\text { The simulation } \\
\text { results(dB) }\end{array}$ & $\begin{array}{l}\text { The theoretical } \\
\text { calculation } \\
\text { values }(\mathrm{dB})\end{array}$ \\
\hline 25 & 24.08 & 23.98 \\
\hline 95 & 29.38 & 29.78 \\
\hline 105 & 25.81 & 25.44
\end{tabular}

The following is two-dimensional matched filtering SNR gain simulation, as shown below, from Fig . 3 can be seen, SNR gain of the two-dimensional matched filtering is a periodic variation along with the noise bandwidth.

Two-dimensional matched filtering is also cyclical changes, the comparing simulation results with theoretical calculations of the two-dimensional matched filter SNR gain are given in table 2 under different noise bandwidth conditions,. from table 3 can be seen, when the noise bandwidth $B_{n}=2 f_{s}-B_{s}=95 \mathrm{MHz}$, the two-dimensional matched filtering SNR gain maximum. This is mainly due to that the Doppler bandwidth and the transmitted signal bandwidth have several orders of magnitude, the impact on SNR is almost negligible.

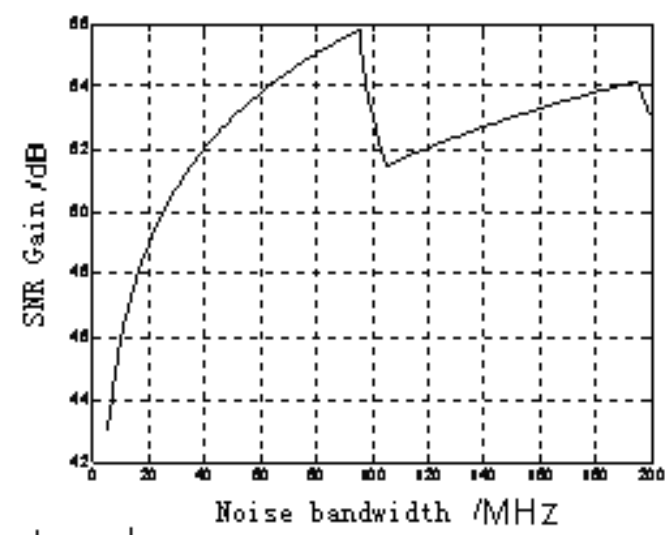

Figure 3. Two-dimensional matched filtering SNR gain and noise bandwidth

TABLE III. Two-DIMENSIONAL MATCHED FILTERING SNR GAIN

\begin{tabular}{lll}
\hline $\begin{array}{c}\text { Noise-bandwidth Bn } \\
(\mathrm{MHz})\end{array}$ & $\begin{array}{l}\text { The-simulation } \\
\text { results }(\mathrm{dB})\end{array}$ & $\begin{array}{l}\text { The theoretical } \\
\text { calculation } \\
\text { values }(\mathrm{dB})\end{array}$ \\
\hline 25 & 49.82 & 50 \\
\hline 95 & 55.36 & 55.8 \\
\hline 105 & 51.81 & 51.46
\end{tabular}

Simulation on the two-dimensional matched filter SNR gain and PRF at the different Doppler bandwidth conditions, from Fig .4 (a) can be seen, the SNR gain increases with increasing PRF, which is coincide with analysis conclusions of formula (30). from a partial enlarged view of Fig .4 (b) can be seen, in a typical SAR system parameters, the SNR gain is positively correlated with the PRF, and the affect of the Doppler bandwidth is small, it can be ignored, which are coincide with analysis conclusions of the formula (30).

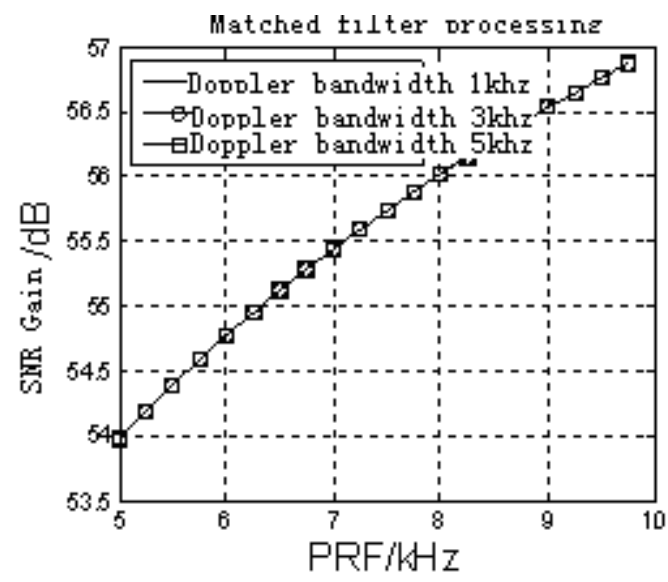

(a) The original figure 


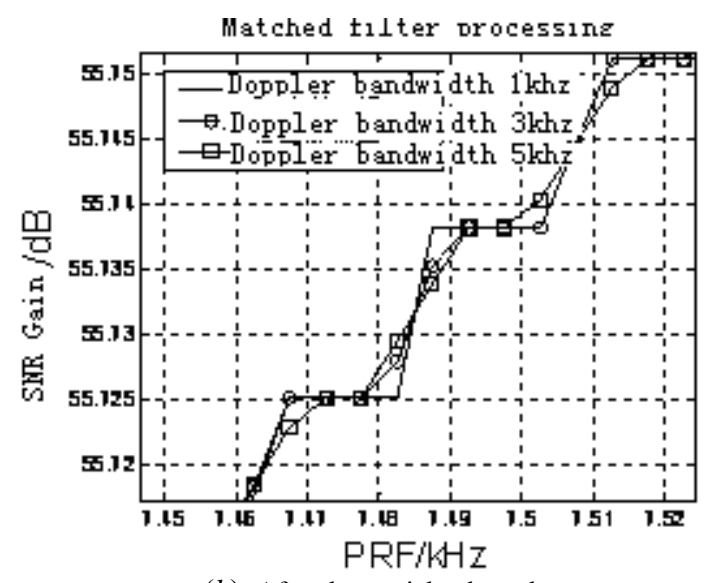

(b) After the partial enlarged

Figure 4. Two-dimensional matched filtering of the relationship between SNR gain and PRF

\section{CONCLUSIONS}

According to the theoretical analysis and simulation results, discrete sampling will lead white noise to colored noise, SNR gain of the two-dimensional matched filters has relationship with noise bandwidth pulse repetition frequency, signal bandwidth, sampling rate in the colored noise conditions, when certain conditions are met, it is equivalent to the traditional matching filter SNR gain. Secondly, SNR gain maxima occur periodically by analyzing the impact of noise bandwidth of SNR under the discrete band-limited white noise conditions, when the sampling rate, the noise bandwidth, signal bandwidth meet the $f_{S}^{*}=\left(B_{n}+B_{s}\right) / 2$, matched filtering SNR gain reaches maximum, signal to noise ratio can be optimized design based on the maximum sampling rate of SAR guidelines.

\section{REFERENCES}

[1] Li Zengliang, Zhao Xin, Ding Zegang. Analysis of diving squint SAR resolution[C]. Proceedings of 2011 IEEE CIE International Conference on Radar, Chengdu, 2011:875-878.

[2] Hu Cheng, Long Teng, Zeng Tao, et al. The accurate focusing and resolution analysis method in geosynchronous SAR[J]. IEEE Transactions on Geoscience and Remote Sensing, 2011, 49(10):3548-3563.

[3] Pi Yiming, Yang jianyu Synthetic aperture radar imaging principle [M]. Chengdu: university of electronic science and technology press, 2007:22 to 30 .

[4] Yuan Xiaokang. Introduction to spaceborne synthetic aperture radar [M]. Beijing: national defence industry press, 2003

[5] QiuXiaoLan chi-biao ding, Hu Donghui. Double SAR imaging processing technology [M]. Beijing: science press, 1993.

[6] ChengLong Jiang, Jiang, Hai, Zhang BingChen, Hong Wen, Wu YiRong. SNR analysis for SAR imaging from raw data via compressed sensing[C]. Proceedings of 9th European Conference on Synthetic Aperture Radar, Hamburg, Germany, 2012:689-692.

[7] ChengLong Jiang, BingChen Zhang, Zhang Z, Hong W, YiRong $\mathrm{Wu}$. Experimental results and analysis of sparse microwave imaging from spaceborne radar raw data[J]. SCIENCE CHINA Information Sciences, 2012, 55(8):1801-1815.

[8] Raney R.K. SNR IN SAR[C]. Proceedings of IEEE IGARSS'85, USA, 1985:994-999.

[9] Wan Yong-lun, Si Qiang, Lu You-xin, Wang Hong, Wang Xuegang. Study of Dechirp Pulse Compression Processing Method for Wideband Signals[J]. Acta Aeronautica et Astronautica Sinica, 2006, 27(5): 917-921.

[10] Wang Xiao-qing, Xiao Jiang, Chen Yong-qiang, Zhu Min-hui. The SNR Study of the Wide Swath SAR Basing on Elevation Multi-receiver[J]. Journal of Electronics \& Information Technology, 2007, 29(9):2101-2104.

[11] Zhang Zhen-hua, Bao Zheng, Xing Meng-dao, Huang Jun-yi. The Analysis of SNR in Passive Distributed Microsatellites SAR System[J]. Journal of Electronics \& Information Technology, 2007, 29(1):15-18.

[12] Curlander J C. Synthetic aperture radar systems and signal processing[M] . New York: John Wiley \& Sons, 1991: 28.

[13] Zhang Jin-dong, Wang Hai-qing, Zhu Xiao-hua, Li Yu-sheng. Adaptive Pulse Compression via MSN Criteria[J]. Journal of Electronics \& Information Technology, 2009,31(4): 790-793.

[14] Zhang Qunying, Yang Xuexian, Han Yueqiu. Relation of Sampling Rate and SNR in Digital Matched Filter System[J]. Systems

[15] Enigeering and Electronics,1999,21(10):63-65. 